\title{
Experimental research of Double-Camera Low Altitude Photogrammetry
}

\author{
Hongcheng Liu \\ National Key Laboratory of Remote Sensing Information \\ and Imagery Analysis, \\ Beijing Research Institute of Uranium Geology \\ Beijing,China \\ liuhckangl@gmail.com
}

\author{
Zongjian Lin \\ Research Center of Surveying and Mapping \\ Chinese Academy of Surveying and Mappin \\ Beijing,China \\ Lincasm@casm.com
}

\begin{abstract}
This paper focus on the design experimentation system of data processing in two camera image, for experimentation of relief stereo mapping and on image processing methods, which base on its composition and dual combination of wide angle digital camera design principles. Practice shows that flight efficiency can be doubled. At last, through experiments the Double Camera Low Altitude Photogrammetry System can achieve 1:1000 scale aerial photographic tasks. As a branch of remote sensing the UAV Borne Double Camera Law Altitude Photogrammetry develop greatly and have a spacious application foreground.

Keywords-low altitude photogrammetr; wide-angle dual combined digital camera; self-calibration
\end{abstract}

\section{INTRODUCTION}

With the rapid development and informationization of economy and society, the demand of high resolution remote sensing image increase every day. How to get fundamental geography data rapidly is a primary problem of our state construction and informationization. As a result of the aviation remote sensing platform and sensor's limit, the aerial photogrammetry is gaining the small area, the big scale data aspect to have the cost to be high, the ratio difference, the elevation cannot achieve questions and so on. Has the low cost and mobile nimble and so on many merit low altitude unmanned aerial vehicle remote sensing can gain the high resolution remote sensing image rapidly in the small area region, is the national aviation remote sensing monitor system important supply, will be the aviation remote sensing future development direction. Owing to the restrietion of platform's volume and weight, UAV can only use single camera system with stable platform presently. Single digital camera's coverage and base-height ratio is small, which increase the interior and field workload, moreover, the elevation accuracy is rather low. So, using Multi-Format mosaic technology, increasing the format size has become a research focus recently.

\section{THE PRINCIPLE OF WIDE-ANGLE AND LIGHT SMALL DUAL COMBINATION DESIGN}

Wide-angle and light small dual combination is use two cameras in the means of inner obliqus to join.Figure 1illustrate the perfect way. The camera's lens to match together. But this way has difficuly at to deal with CCD connect and spectroscope to fix. Presently the aerial aerial camera use the method of outer obliqus to join in general.The case in point is
DMC. UltraCamD use the two ways.In the way of outter obliqus, owing to every camera has different size, the camera has height displacement result in projection focal can't overlap.

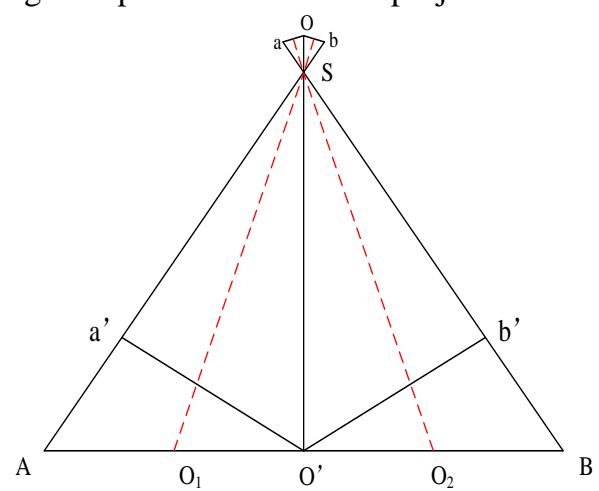

Figure 1. Perfect two canmera mosaic model

So, we can design the structure as So, we can design the structure asfigure2 illustrate. In this way, two camera to slope the same tumble angle $\omega$, thus to make up the expand the view scope angle A1SA2 and to make up the overlap region B1SB2. We can conduct self-calibration with the help of the overlap region to confirm the origin distortion.

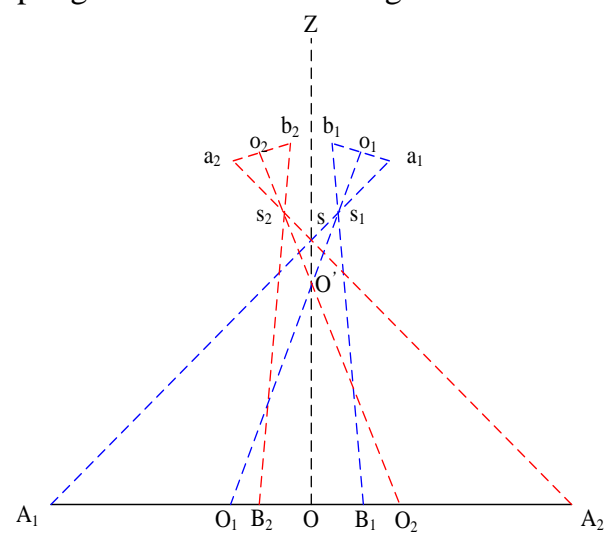

Figure 2. Field of view mosaic model with overlay

Actualy, wide-angle and light small dual combination of low-altitude aerial camera system design the structure which illustrate in Figure3. two camera fly along the airline to take a picture. The first camera to incline left $\omega$ angle, the second 
camera to incline right $\omega$ angle. In advance, accurate measure the two cameras themselves later photograph time by an oscilloscope. Consider the origin later photograph time in the synchronism control photograph structur.and the compensate later photograph time which inverse compute in accordance with the diatance of two camera in the fly dieection. We can design the the synchronism control photograph structure later time and can make the contemporary photograph probably in this way. The influence of the surplus time error can eliminate by the way of self-calibration in later.

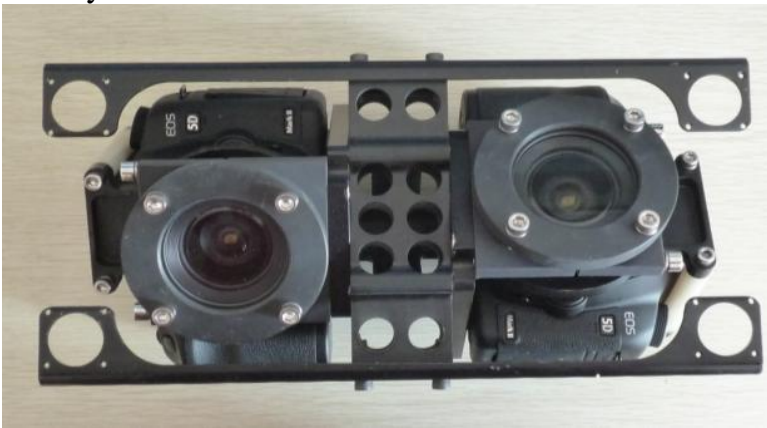

Figure 3. Two combined-camera system

\section{THE SUMMARY OF THE EXPERIMENTATION}

\section{A. The general situation of the experimential region}

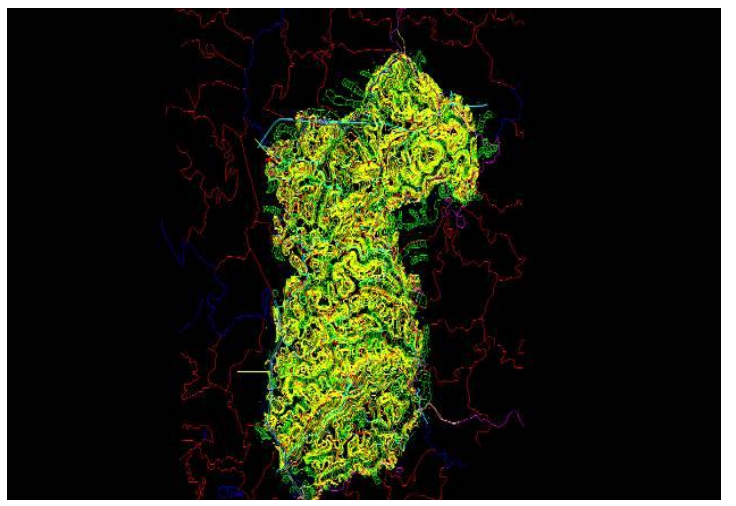

Figure 4. The relief map of experimential region

The experimential region is a mountainous region. The highest region is 323 miters. The lowest region is 323 miters. The terrain has the average slope about 6 . Figure 4 is the relief map.Figure 5 is the map of contour line.

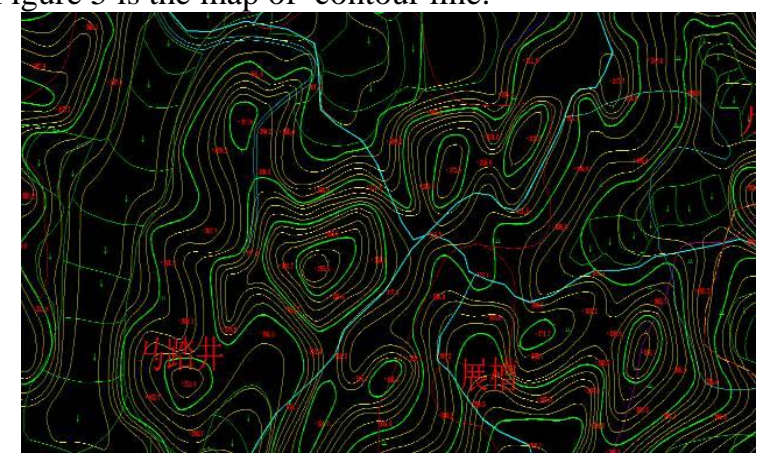

Figure 5. The map of contour line

\section{B. The airline design of the experimential region}

The experimental area about 15 kilometers. This flying height is 500 metres and gain 1006 images. The resolving power of image is 0.1 meters. Aireline design map for experiment is figure 6.

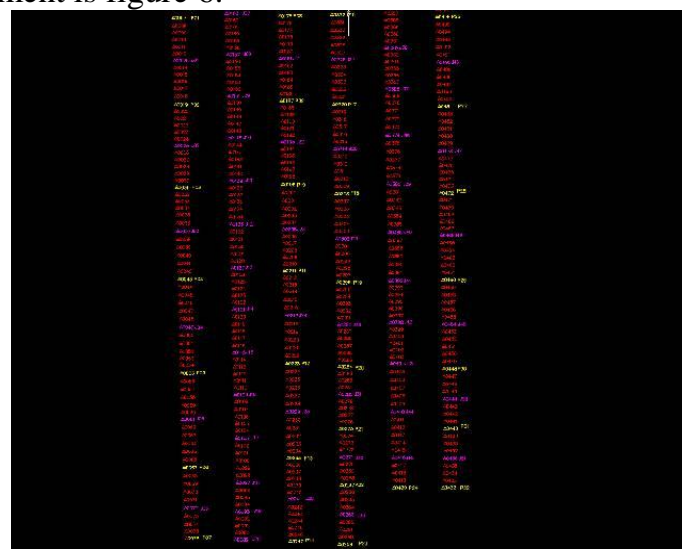

Figure 6. Aireline design map for experiment

\section{Ground point measuring}

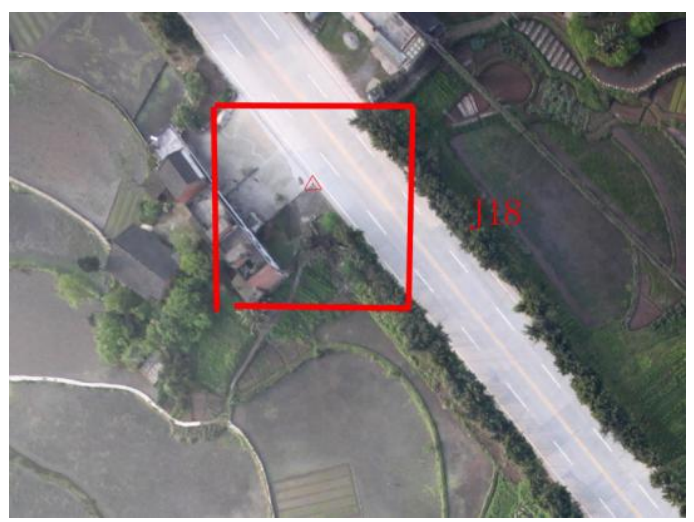

Figure 7. Check Point Location

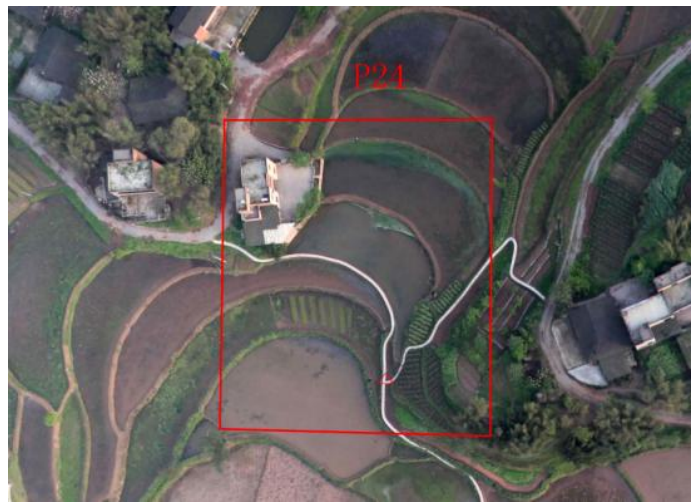

Figure 8. Control Point Location

We can choose the apparent feature in some images. The control point had better in the center of the image and the terrain is flat. In the interval of airelines maybe choose a aireline,in which choose the ground control point. In the interval of images maybe choose an image, in which choose 
the check point and the ground control point.Choose 32 ground control points and 51 check points in all in the region. Figure 6 is the Image control points layout picture.

\section{THE CALIBRATION AND MOSAIC OF TWO COMBINED- CAMERA}

\section{A. The calibration of Two combined-camera}

Combined camera calibration is same as the general aerial photography camera calibration. Every camera use inner orientation and distortion correetion equation estimate camera parameters through to photograph the outdoor or indoor calibration field.

$$
\left.\begin{array}{l}
x-x_{0}+\Delta x=-f \frac{a_{1}\left(X-X_{s}\right)+b_{1}\left(Y-Y_{s}\right)+c_{1}\left(Z-Z_{s}\right)}{a_{2}\left(X-X_{s}\right)+b_{2}\left(Y-Y_{s}\right)+c_{2}\left(Z-Z_{s}\right)} \\
y-y_{0}+\Delta y=-f \frac{a_{3}\left(X-X_{s}\right)+b_{3}\left(Y-Y_{s}\right)+c_{3}\left(Z-Z_{s}\right)}{a_{2}\left(X-X_{s}\right)+b_{2}\left(Y-Y_{s}\right)+c_{2}\left(Z-Z_{s}\right)}
\end{array}\right\}
$$

In which $\left(x_{0}, y_{0}\right)$ is the internal orientation, $\mathrm{f}$ is the focual length, $\Delta x, \Delta y$ is the distortion 。

$$
\begin{aligned}
& \Delta x=\left(x-x_{0}\right)\left(k_{1} r^{2}+k_{2} r^{4}\right)+ \\
& p_{1}\left[r^{2}+2\left(x-x_{0}\right)^{2}\right]+2 p_{2}\left(x-x_{0}\right)\left(y-y_{0}\right) \\
& +\alpha\left(x-x_{0}\right)+\beta\left(y-y_{0}\right) \\
& \Delta y=\left(y-y_{0}\right)\left(k_{1} r^{2}+k_{2} r^{4}\right)+p_{2}\left[r^{2}+2\left(y-y_{0}\right)^{2}\right] \\
& +2 p_{1}\left(x-x_{0}\right)\left(y-y_{0}\right) \\
& r=\sqrt{\left(x-x_{0}\right)^{2}+\left(y-y_{0}\right)^{2}}
\end{aligned}
$$

In which, $r$ is the distance between image point and the center. $k_{0}, k_{1}, k_{2}$ is the radial distortion coefficient, $p_{1}, p_{2}$ is the tangential distortion coefficient.We can estimate the relative orientation parameter through Space resection,and then translate to combined camera calibration the coordinate system which use two-camera center projection line for virtual projection line. The calibration result illstruate in table 1.

TABLE I. TWO-CAMERA SYSTEM CALIBRATION RESULT

\begin{tabular}{|c|c|c|c|}
\hline & Front Camera & Back Camera & VirtualCamer \\
\hline Focal Length & 3795.0126 & 3792.7652 & 3800 \\
\hline Image Width & 5616 & 5616 & 9856 \\
\hline Image Height & 3744 & 3744 & 3648 \\
\hline A1 & $7.89491 \mathrm{E}-09$ & $7.91905 \mathrm{E}-09$ & 0 \\
\hline A2 & $-5.35 \mathrm{E}-16$ & $-5.32 \mathrm{E}-16$ & 0 \\
\hline P1 & $1.5366 \mathrm{E}-07$ & $-1.101 \mathrm{E}-08$ & 0 \\
\hline P2 & $-3.52809 \mathrm{E}-08$ & $-9.16327 \mathrm{E}-08$ & 0 \\
\hline C1 & -0.000281277 & 0.000337663 & 0 \\
\hline C2 & 0.00015474 & $-9.02062 \mathrm{E}-05$ & 0 \\
\hline
\end{tabular}

\section{B. The mosaic of Two combined-camera}

If elements of relative orientation has no error through analysis the overlay region and two camera exposure synchronously, the overlap region would be match together perfectly. But due to these two errors exist, the overlap region would be not match together. We can search out the parallax and vertical parallax cause by two images are not overlay in the characteristic through image matching. Then, we can estimate the remaining error by means of least squares correlation.

$$
\left\{\begin{array}{l}
\Delta x=\Delta b_{x}+\frac{x}{H} \Delta b_{z}+H\left(1+\frac{x^{2}}{H^{2}}\right) \Delta \varphi+\frac{x y}{H} \Delta \omega-y \Delta \kappa \\
\Delta y=\Delta b_{y}+\frac{y}{H} \Delta b_{z}+\frac{x y}{H} \Delta \varphi+H\left(1+\frac{y^{2}}{H}\right) \Delta \omega+x \Delta \kappa
\end{array}\right.
$$

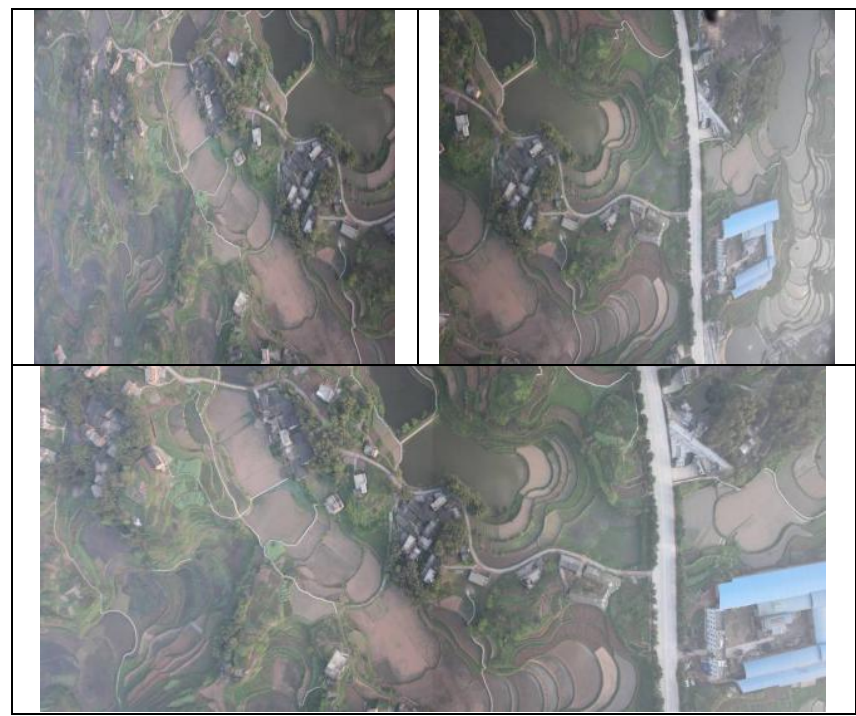

Figure 9. Two- Camera Image and Virtual Image

Where $\mathrm{x}$ and $\mathrm{y}$ is known as the coordinate in which the origin coordinate is the principal point of photograph in the virtual image. $\mathrm{H}$ is the distance which between projection plane and projection center. Generally speaking, $\mathrm{H}$ maybe defined as $H=\frac{1}{2}\left(f_{1}+f_{2}\right)$.In which $f_{1}, f_{2}$ is the focal length. $\Delta b_{x}, \Delta b_{y}, \Delta b_{z}, \Delta \varphi, \Delta \omega, \Delta \kappa$ is the remaining error of relative orientation and approximate value. We can eliminate paralla. The virtual image and photo are shown in Figure 9.

\section{THE PROGESS OF AIR TRIANGULATION}

\section{A. Feature extraction in stereo photopair}
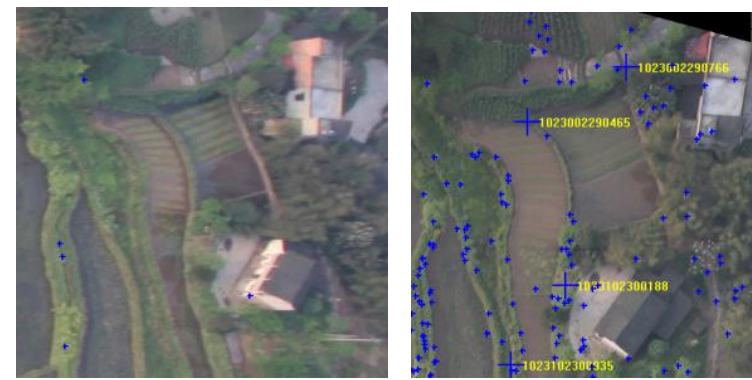

Figure 10. Feature extraction in stereo photopair

In the edge of the image, there are many deformations, as shown in figure 10 , the corresponding image points has 
many difference in the stereo photopair. So, we must be as less as possible to extract the feature point in these region.

\section{B. The error of relative orientation progess}

We can estimate relative orientation parameters and extract the feature points in the relative orientation progess. Then, we can estimate the mean square error through block adjustment he result as shown in Table 2.

\section{Region adjustment}

We can choose the standard airline that fly height is stable from all aielines, then control other airelines in proper order use the control point in the among aireline model. At last estimate the MSE of the join point in the region adjustment process. The result of the coordinate MSE is $0.137,0.214$, 0.418 .

\section{Use GCP for absolute orientation}

We can adjust the four ground control points be distributed over the region to the accurate location, then shear the image which other control point exist.which illstrute in Figure 11.At last estimate the coordinate MSE of the GCP is $0.143,0.305$, 0.495 .

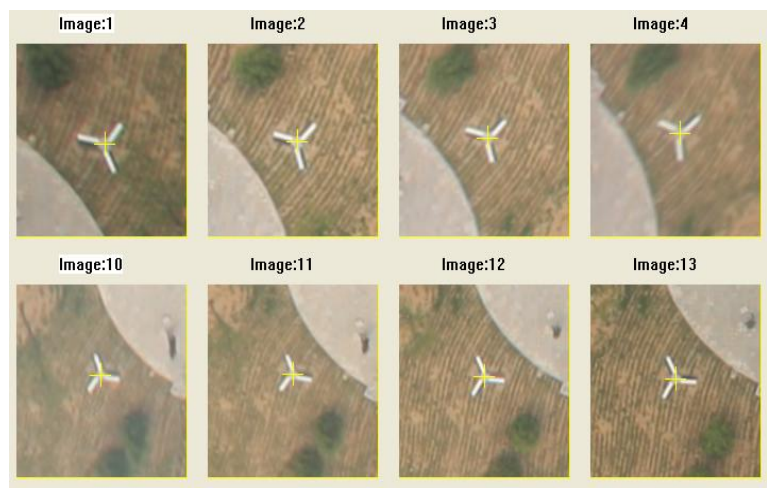

Figure 11. The Adjustion of the GCP

\section{CONCLUSION}

Through experiments the paper proved that, wide-angle and light small dual combination of low-altitude aerial camera system can achieve 1:1000 scale aerial Photographic tasks.Due to the width parallel the flying direction, baseheight ratio is short and the height precision is low. So, we can attempt the height parallel the flying direction, enhance the base-height ratio so that improve the height precision.

\section{References}

[1] Lin Zongjian, Su Guozhong, ZHI Xiaodong. UAV Borne Double Camera Law Altitude Photogrammetry System:Geospatial Information, Vol 243, pp.1-3, April 2010 (In Chinese)

[2] Gou Zhiyang, Zhao Hongying, Yan Lei. Borne.Aerial Photogrammetric Quality Evaluation of the UAV: Image Technology, ,pp.49-51, February 2007 (In Chinese)

[3] Jia Jianjun, Shu Rong, Wang Binyong. Large frame CCD camera system for unmanned aircraft remote sensing: OptoElectronic Engineering, Vol 33, pp.90-93, Auguest 2006 (In Chinese)
[4] Zhang Zuxun.:Digital Photogrammetry and Computer Vision: Geomatics and Information Science of Wuhan University, Vo 29, pp.1035-1039, December 2004 (In Chinese)

[5] Su Guozhong, ZHANG Zuxun. How to Relate the openGL Imaging Process with Exterior and Interior Parameters of PhotOgrammetry: Joumal of Image and Graphics, Vol 11, pp.540543, April 2006 (In Chinese)

[6] Lu Hongwei, Qiu Zhiqiang. Photogrammetry Based on the Image Sequence: Image Technology Acquired by the Satellite Formation Journal of National University of Defense Technology , Vol 26, pp.1618, February 2007 (In Chinese)

[7] Zhang Jianxia, Wang Liuzhao, Jiang Jinbao. Low-altitude Aerial Survey Research Based On Digital Image:Bulletin of Surveying and Mapping, pp.29-32, November 2006 (In Chinese)

[8] Zhang Zuxun.,Yang Shengchun,Zhang Jianqing: Multi-Baseline Digita Close-Range Photogrammetry:Geospatial Information, Vol 5, pp.1-5, February 2007 (In Chinese) 